\title{
La complainte du proctologue...
}

\section{F. Devulder \\ (C) Springer-Verlag France 2011}

Si l'on en croit le programme de la Journée de printemps de la Fédération des spécialités médicales (FSM) qui se déroulera le 26 mai prochain à l'hôpital d'instruction des armées du Val-de-Grâce, sous le haut patronage de Xavier Bertrand, ministre du Travail, de l'Emploi et de la Santé, le développement professionnel continu (DPC), c'est pour demain... Cela risque d'en faire sourire plus d'un! Voilà en effet plus de 15 ans que notre formation médicale continue (FMC) erre entre les hésitations de notre tutelle. C'est en effet avec les ordonnances Juppé de 1996, puis la loi du 4 mars 2002 et le code de santé publique du mois d'août 2004 qu'apparaissait la FMC créditante. On était alors soumis à une obligation quinquennale de FMC. À l'instar des autres pays européens et nord-américains, nous devions glaner des points de formation. À cette FMC créditante s'est adjointe, avec la loi portant réforme de l'assurance maladie du 13 août 2004, une obligation d'évaluation des pratiques professionnelles (EPP) sous le contrôle rigoureux de la Haute Autorité de santé (HAS). Ce système était donc en place. Nombre d'organismes de formation se sont alors engouffrés dans des programmes d'EPP. Les congrès valaient des points, les revues aussi... Un livret était même établi pour faciliter le travail de chacun pour répondre à ces obligations qui devaient être validées auprès des conseils régionaux de FMC (CRFMC). Qui « devaient », en effet, car dès sa nomination, Mme Roselyne Bachelot Narquin, ministre de la Santé, de la Jeunesse et des Sports, enterrait les CRFMC et rendait tout ce dispositif caduc. L'heure du DPC était née, officialisée avec l'article 59 de la loi « Hôpital, patient, santé et territoire » dite loi HPST. D'après cet article 59, le DPC est une obligation pour tous les médecins. Inspiré des systèmes anglo-saxons et nord-américains, ce DPC a pour ambition de mettre en musique FMC et EPP, avec une touche de maîtrise médicalisée et un zeste de sécurité des pratiques. Ambitieux diront les uns. Séduisant diront les autres. Fumisterie diront les grincheux. Inapplicable en l'état subodoreront la plupart. Force est de constater qu'il est difficile de ne pas donner raison à ces derniers qui avaient vu juste lorsque l'on nous assénait que le DPC serait effectif pour tous dès le début

F. Devulder $(\bowtie)$

Polyclinique de Courlancy, 38, rue de Courlancy,

F-51100 Reims, France

e-mail : franckdevulder@gmail.com de cette année ! En effet, le DPC a du retard. Notre tutelle avait en effet, entre autres, quasiment exclu les médecins du dispositif de gestion de leur DPC, confié à l'État et à l'assurance maladie. Il n'en fallait pas moins pour unir ordre des médecins, conférence nationale des universités et syndicats médicaux pour s'opposer d'une seule voix à cette offense qui nous était faite. Après des mois de tergiversations et, au passage, un changement de locataire au ministère de la Santé, nous voici donc sur de bons rails ou presque... Effet, Médiator $^{\circledR}$ ou pas, le ministère tarde aujourd'hui à mettre en place ce dispositif dont le financement n'est pas totalement réglé. On nous annonce la sortie des décrets pour septembre 2011, ce qui se traduirait par un DPC en 2012, mais une véritable obligation en 2013. Qui croire ? Xavier Bertrand prendra-t-il le risque de décevoir les médecins à quelques mois d'une échéance électorale majeure?

On le voit bien. Le DPC, en introduisant une amélioration des pratiques médicales selon le principe de la roue de Deming, est certainement séduisant. Cependant, les vraies questions nous ont-elles été posées ? Notre tutelle a-t-elle cherché à comprendre ce qui faisait la qualité de « notre » médecine ? La première pierre d'un DPC qui mettra des années avant d'entrer réellement dans notre pratique n'estelle pas la FMC ? Les lecteurs assidus de Côlon \& Rectum se délecteront ce mois-ci d'une mise au point consacrée au traitement des fissures anales, d'une autre sur la prise en charge des rectopexies en 2011 ou prendront de la hauteur en découvrant le passionnant mano a mano sur l'art de combattre l'iléite de Crohn. Et finalement, si c'était là la voix de la médecine de qualité ! Il suffirait pour s'en convaincre de constater la fréquentation sans cesse grandissante des journées de coloproctologie, du congrès de l'AFC ou des JFHOD, pour ne citer que les principales grand-messes hexagonales. Invitons nos décideurs à rejoindre les ambiances studieuses des coloproctologues passionnés qui, congrès après congrès, font avancer la médecine et améliorent la prise en charge des patients. Chacun, par l'étude de revues de qualité, par l'enseignement au sein de DIU ou par les richesses du compagnonnage, participe déjà au DPC de... demain! Notre voix doit être entendue afin que le DPC voulu par l'État, et probablement souhaité par la population, s'inscrive dans la continuité de nos nombreuses années consacrées à l'amélioration de nos pratiques. 